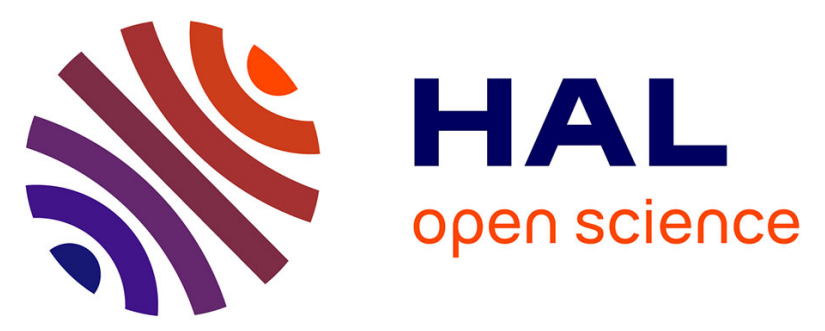

\title{
Faucon Noir : retour d'expérience sur une étude de la biodiversité par drone
}

\author{
Laurent Beaudoin, Antoine Gademer, Loïca Avanthey, Samira Mobaied, \\ Jean-Paul Rudant
}

\section{- To cite this version:}

Laurent Beaudoin, Antoine Gademer, Loïca Avanthey, Samira Mobaied, Jean-Paul Rudant. Faucon Noir : retour d'expérience sur une étude de la biodiversité par drone. Colloque scientifique francophone Drones et moyens légers aéroportés d'observation, Jun 2014, Montpellier, France. hal-01132264

\section{HAL Id: hal-01132264 https://hal.science/hal-01132264}

Submitted on 16 Mar 2015

HAL is a multi-disciplinary open access archive for the deposit and dissemination of scientific research documents, whether they are published or not. The documents may come from teaching and research institutions in France or abroad, or from public or private research centers.
L'archive ouverte pluridisciplinaire HAL, est destinée au dépôt et à la diffusion de documents scientifiques de niveau recherche, publiés ou non, émanant des établissements d'enseignement et de recherche français ou étrangers, des laboratoires publics ou privés. 
Faucon Noir : retour d'expérience sur une étude de la biodiversité par drone

(2)

L. Beaudoin ${ }^{\dagger}$

A. Gademer ${ }^{\dagger}$

L. Avanthey ${ }^{\dagger, \ddagger}$

S. Mobaied \#

J.P. Rudant ${ }^{\S}$

${ }^{\S}$ Laboratoire Géomatique, Télédétection, Modélisation des Connaissances (GTMC), Université Paris-Est, France, contact: rudant@univ-mlv.fr †Pôle Acquisition et Traitement des Images et des Signaux(ATIS), ESIEA Recherche, France, contact: beaudoin@esiea.fr

‡ Département Traitement des Images et du Signal(TSI), Telecom-ParisTech, France \# Département Écologie Gestion de la Biodiversité, CNRS-MNHN, France

L'étude de la biodiversité et de la dynamique des écosystèmes est un enjeu majeur dans le cadre de la gestion des réserves

biologiques. Pour cela, il est indispensable de réaliser une cartographie très précise, si possible à l'échelle des individus. Nous

présentons ici notre retour d'expérience sur l'utilisation d'un drone pour réaliser cette tâche, aujourd'hui effectuée manuellement.

\section{Gestion des réserves biologiques}

La zone d'étude de la « Mare aux Joncs » est située dans la Réserve de Biosphère (MAB) du site de Fontainebleau. Ce site contient deux habitats d'intérêt communautaire (Natura 2000) qui hébergent des espèces très particulières : la lande sèche et la lande humide.
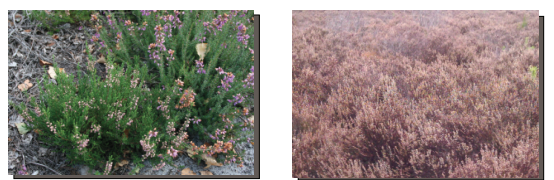

Deux dangers menacent ces espèces

- la reforestation
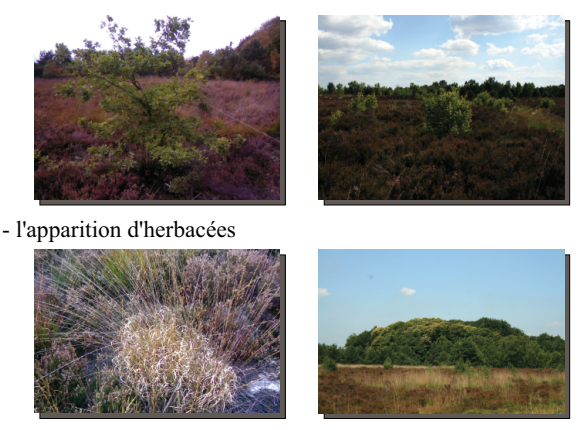

- l'apparition d'herbacées
Cartographie à l'échelle de l'individu Pratique courante : cartographie métrique.

$\sim 600$ individus $(\varnothing>10 \mathrm{~cm})$

Occupation des sols et mesure des pentes.

Mesures de la dynamique d'évolution de l'écosystème.

Besoin d'un système flexible (« à la demande »).

Besoin de précision dans l'identification et la caractérisation des espèces

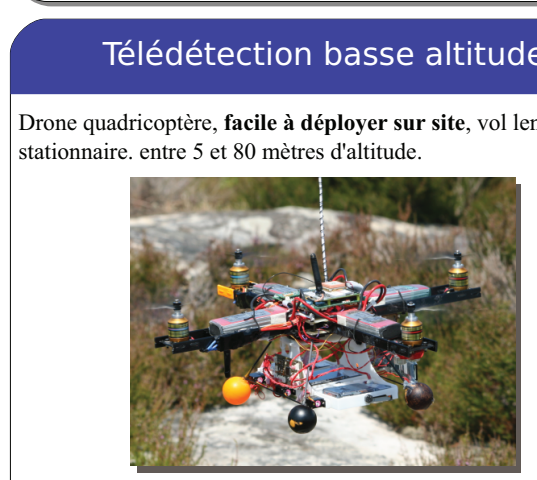

Monitoring complet depuis la station sol, retour vidéo temps réel et contrôle direct de la charge utile. manuelle de la zone selon un pas

Drone quadricoptère Faucon Noir

$\begin{array}{ll}\text { Taille : } & \mathbf{7 0} \mathbf{~ c m} \\ \text { Poids : } & \mathbf{1 . 6} \mathbf{~ k g} \\ \text { Puissance : } & 1 \mathrm{~kW} \\ \text { Charge utile : } & \mathbf{1 ~} \mathbf{~ k g} \\ \text { Hauteur de vol : } & 80 \mathrm{~m} \text { (vue directe) } \\ \text { Endurance : } & 25 \mathrm{~min} \text { (à vide) } \\ \text { Géolocalisation : } & \text { GPS \& AHRS } \\ & \\ 3^{\text {iẻme }} \text { au Challenge } & \text { Minidrones de l'ONERA et la DGA }\end{array}$

Capteur tri-caméras synchronisées

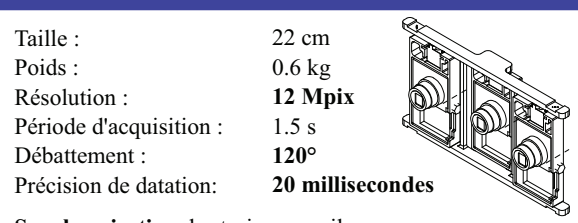

Synchronisation des trois appareils.

Contrôle électronique de l'allumage, du déclenchement et de la datation à la milliseconde des images.

Modularité : orientation stéréoscopique ou oblique.

\section{Liaisons de communication}

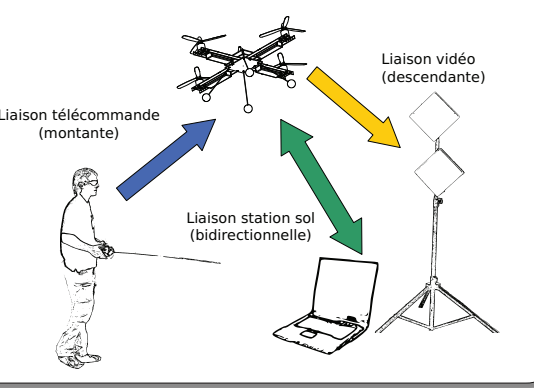

\section{Reconstruction 3D}

Pour chaque couple, une surface 3D est calculée comme suit - détection de points d'intérêt : SURF

- estimation de la matrice fondamentale : détection d'outliers avec RANSAC,

Des outils pertinents pour la gestion de la biodiversité

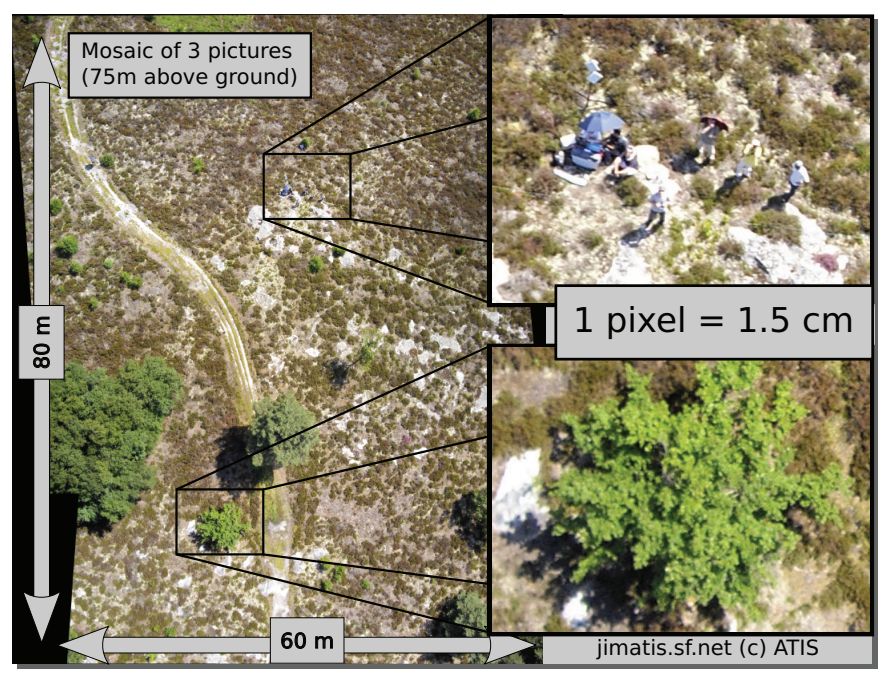

- Haute résolution permet de distinguer les individus et leurs caractéristiques (forme et taille des feuilles, densité et composition des buissons mixtes, etc.)

Triplets d'images pour différents usages : couples stéréoscopiques pour la reconstruction 3D , amélioration du calcul de la biomasse, images obliques pour une meilleure caractérisation des hauteurs et des formes.

- L'utilisation des micro-drones permet couverture de zones relativement arges sans limite de temps ou revisite ce qui permet l'utilisation des propriétés phénologiques des plantes.

Contrôle direct par les thématiciens, ce qui ouvre des possibilités en termes de gestion et de conservation de la biodiversité.
- rectification en géométrie épipolaire : réduction des distortions grâce à une rectification épipolaire polaire.

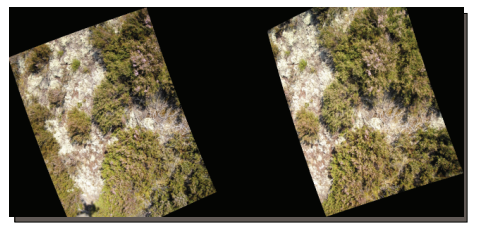

- Appariement dense avec un appariement hiérarchique robuste. On obtient une carte de disparité dense.

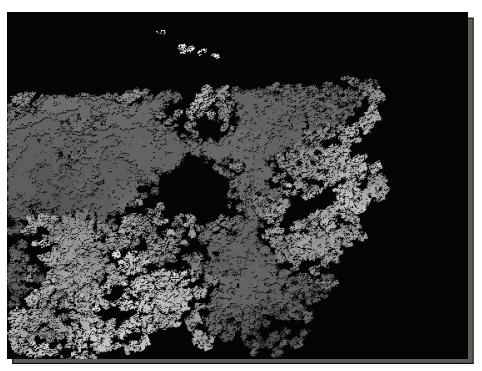

